\title{
Does Rivaroxaban have a fibrinolytic effect?
}

\author{
PL Antignani ${ }^{1}$, C Allegra ${ }^{2}$ \\ ${ }^{1}$ Director, Vascular Center, Nuova Villa Claudia, Rome, Italy \\ ${ }^{1}$ Founding President, Central European Vascular Forum
}

submitted: Mar 25, 2018, accepted: Mar 26, 2018, EPub Ahead of Print: Mar 28, 2018, published: Jun 30, 2018

Conflict of interest: None

DOI: 10.24019/jtavr.46 - Corresponding author: Prof. Pier Luigi Antignani, antignanipl@gmail.com

(C) 2017 Fondazione Vasculab impresa sociale ONLUS. All rights reserved.

\begin{abstract}
Aim

The study started from the observation of complete recanalization of thrombus in subjects with DVT treated with rivaroxaban after 1-2 weeks. Aim of this clinicalinstrumental observational retrospective research is to evaluate clinically and by means of Echo color Duplex the fibrinolytic effect of Rivaroxaban in patients with recent and previous DVT.

Methods

We evaluated two populations of patients:

$1^{\text {st }}$ group: 27 patients males (range of age 50-73 years) with popliteal-femoral DVT (12 months before) treated with standard anticoagulant therapy. In this patients we found a complete superficial femoral recanalization and partial recanalization of the popliteal vein $(30 \%$ of residual thrombus). The patients had normal creatinine clearance and liver function. The patients switched from warfarin to rivaroxaban for no compliance to warfarin.

$2^{\text {nd }}$ group: 19 patients (range of age 65-85 years) with previous popliteal-femoral DVT and complete common femoral veins recanalization (already know or
\end{abstract}

\section{Introduction}

The objectives of anti-coagulation are prevention of extension of the thrombus, avoidance of pulmonary embolism (PE), prevention of recurrence of VTE and ultimately the avoidance of post-thrombotic syndrome (PTS). Considerable progress in the treatment of deep-vein thrombosis (DVT) of the lower extremities was achieved with the development of direct non-vitamin $\mathrm{K}$ antagonists (DOACs) and fibrinolytic therapies. Rivaroxaban is an oral, direct factor Xa inhibitor that inhibits free and clot-bound documented). The patients presented recent superficial femoral vein re-thrombosis (1 week before). The patients had normal creatinine clearance and liver function.

Results

$1^{\text {st }}$ group: we observed in all patients the complete recanalization of the popliteal veins.

$2^{\text {nd }}$ group: we observed in all patients the complete recanalization of the superficial femoral veins after 2 weeks of the therapy of Rivaroxaban and complete recanalization of the popliteal veins after 4 weeks. No adverse events for both groups were observed.

Conclusion

According to our results, we consider that Rivaroxaban could have a fibrinolytic effect not only on recent thrombus but also on organized thrombus with, as result, a complete recanalization of affected veins and consequent reduction of incidence of post-thrombotic syndrome saving the valves structure.

Keywords Acute venous thrombosis, re-thrombosis, DOACs, post-thrombotic syndrome, fibrinolysis

factor Xa and factor Xa in the prothrombinase complex. Preclinical studies demonstrated a potent anticoagulant effect of rivaroxaban in plasma as well as the ability of this agent to prevent and treat venous and arterial thrombosis in animal models. These studies led to an extensive phase I clinical development program that investigated the pharmacological properties of rivaroxaban in humans ${ }^{1,2,3}$.

The pharmacodynamic effects of rivaroxaban (for example, inhibition of factor $\mathrm{Xa}$ and prolongation of 
prothrombin time) were closely correlated with rivaroxaban concentrations in plasma ${ }^{4}$.

Rivaroxaban is indicated for the treatment of deep vein thrombosis (DVT), and prevention of recurrent DVT and pulmonary embolism (PE) following an acute DVT in adults. For the initial treatment of acute deep vein thrombosis, the recommended dosage of rivaroxaban is $15 \mathrm{mg}$ twice daily for the first 21 days followed by 20 $\mathrm{mg}$ once daily for continued treatment and prevention of recurrence $\mathrm{e}^{5-8}$.

During the treatment of the patients with DVT we observed the complete recanalization of thrombus after 1-2 weeks. We presented these results during some congresses and other authors reported the same observation.

Aim of this clinical-instrumental observational retrospective research is to evaluate clinically the fibrinolytic effect of Rivaroxaban in patients with recent and previous DVT, i.e.with re-thrombosis or organized thrombus.

\section{Material and methods}

We evaluated two populations of patients (see table I)

$1^{\text {st }}$ group: 27 patients (mean age $50-73$ years) with previous popliteal-femoral DVT (max 12 months before) treated with standard anticoagulant therapy at long time for different reasons.

The patients switched from warfarin to rivaroxaban for no compliance to warfarin (frequent long distance travel with important jet-lag, no observation for the food, continuous change of INR values, no correct observance of dosages).

At the first observation (time 0) by means of echo duplex we found a complete superficial femoral recanalization and partial recanalization of the popliteal vein (with $30 \%$ of residual thrombus).

$2^{\text {nd }}$ group: 19 patients (mean age 65-85 years) with the same presentation of $1^{\text {st }}$ group (previous poplitealfemoral DVT with the complete common femoral veins recanalization and partial popliteal recanalization and residual thrombosis of $30 \%$, already know or documented) but with recent superficial femoral vein re-thrombosis (onset 1 week before).

In these patients we started with the treatment with rivaroxaban due to intolerance to low molecular weight heparin (L.M.W.H.) and no compliance to warfarin.

We treated all patients with $15 \mathrm{mg}$ BID for 21 days followed by $20 \mathrm{mg}$ OD of Rivaroxaban for 3 months. All patients had normal creatinine clearance and liver function.
Both groups were submitted to the clinical and echo duplex examination at time 0 and at 1,2,3,4 weeks and after 3 months during the DOACs treatment.

\section{Results}

$1^{\text {st }}$ group: we observed in all patients after 2 weeks the complete recanalization of the popliteal veins (previous thrombosis with $30 \%$ of residual thrombus).

$2^{\text {nd }}$ group: in the same way, we observed in all patients the complete recanalization of the popliteal veins (previous thrombosis) after 4 weeks.and the complete recanalization of the recent thrombosis of the superficial femoral veins after 2 weeks of the therapy of Rivaroxaban.

No adverse events for both groups were observed.

\section{Conclusions}

Factor $\mathrm{X}$ has long been known to have a key role in hemostasis, and its activated form, factor $\mathrm{Xa}$, has a significant function in the blood coagulation pathway because it catalyzes the production of thrombin, which leads to clot formation. Activation of factor $\mathrm{X}$ to factor $\mathrm{Xa}$ occurs through both the intrinsic and extrinsic pathways of the coagulation cascade. Factor $\mathrm{Xa}$ initiates the final, common pathway that results in thrombin activation via the prothrombinase complex. Early studies of naturally occurring factor Xa inhibitors indicated that targeting factor $\mathrm{Xa}$ could provide effective anticoagulation $^{8}$. Selective inhibition of factor Xa produces antithrombotic effects by decreasing the generation of thrombin, thus diminishing thrombin-mediated activation of both coagulation and platelets without affecting the activity of existing thrombin ${ }^{9,10}$.

In the paper of Garabon ${ }^{11}$ Rivaroxaban was shown to decrease the rate of coagulation, which was decreased further at higher concentrations of thrombomodulin (TM). The presence of either thrombin activatable fibrinolysis inhibitor (TAFI) variant also seemed to decrease clot formation rate at higher levels of rivaroxaban and TM. At all concentrations of TM, TAFI-dependent resistance to fibrinolysis was attenuated by rivaroxaban. The effect of rivaroxaban was, however, greater for wild-type TAFI than for the T325I variant. In conclusion, rivaroxaban exhibits TAFI-dependent profibrinolytic effects that are influenced by the levels of TM and the by intrinsic stability of TAFIa. TM also affected the dynamics of coagulation. These findings suggest a role for the anatomical location of a procoagulant stimulus and plasma TM-altering disease phenotypes in the pharmacodynamics and a role for the T325I polymorphism in the pharmacogenomics of rivaroxaban. 
$1^{\text {st }}$ group

Patients

Mean age

Range

Males/females

Onset of previous thrombosis

Onset of acute thrombosis

Complications

Adverse effects by rivaroxaban
27

61,5 years

50-73 years

$9 / 18$

max 12 months

...

$\max 12$ months

none

none
1 week

none

$2^{\text {nd }}$ group

19

66,8 years

65-85 years

$8 / 11$

none

Table I - Patients data subdivided by groups. Treatment: rivaroxaban $15 \mathrm{mg}$ BID for 21 days followed by $20 \mathrm{mg}$ OD for 3 months. BID - bis in die. OD - once a day.

Álvarez ${ }^{12}$ also showed that Rivaroxaban also altered ( $>2$ fold change) the expression of matrix metallopeptidase 2 and urokinase plasminogen activator (u-PA), but counteracted the FXa $(9 \mathrm{nM})$-induced up-regulation of several pro-inflammatory genes $(\mathrm{P}<0.05)$ and FXaenhanced platelet adhesion over human umbilical vein endothelial cells (HUVEC). Rivaroxaban increased u-PA protein expression in HUVEC supernatants and enhanced u-PA activity (up to $4 \mathrm{IU} \mathrm{ng}^{-1}$ of u-PA). Rivaroxaban ( $1 \mathrm{nM}-$ $1 \mu \mathrm{M})$ showed a significant and dose-dependent positive effect on HUVEC growth that was inhibited by BC-11hydroxibromide, an inhibitor of u-PA. Healing properties after a wound on HUVEC cultures, and fibrinolytic properties were also shown by rivaroxaban. Both effects were reversed by BC-11-hydroxibromide. Rivaroxaban enhanced viability, growth and migration of HUVEC, mainly by u-PA activation and upregulation, which also participate in the rivaroxaban-induced fibrinolytic activity at endothelial level. Rivaroxaban also protected from the pro-inflammatory effects of FXa on HUVEC. Altogether may improve endothelial functionality and could contribute to the cardiovascular benefits of rivaroxaban.

From the clinical point of view, the EINSTEIN investigators ${ }^{13,14}$ conducted a post-hoc subgroup analysis of the Einstein DVT trial $(\mathrm{N}=3449)$ to assess the impact of rivaroxaban therapy on the development of PTS. KaplanMeier survival analysis was performed to compare the cumulative incidence of PTS between the rivaroxaban and enoxaparin/VKAgroups. They included 336 patients, $162(48 \%)$ treated with rivaroxaban and $174(52 \%)$ with enoxaparin/VKA. The cumulative PTS incidence at 60 months was $29 \%$ in the rivaroxaban group and $40 \%$ in the enoxaparin/VKAgroup. After adjusting for age, gender, body mass index, previous VTE, ipsilateral recurrent DVT, extent of DVT, idiopathic DVT, duration of anticoagulant treatment, compliance to assigned study medication, elastic compression stocking use and active malignancy, the HR of PTS development for rivaroxaban was 0.76 (95\% CI: 0.51-1.13). The investigators concluded that treatment of acute DVT with rivaroxaban was associated with a numerically lower but statistically non-significant reduction in risk of PTS compared with enoxaparin/ VKAtreatment.

Van $\mathrm{Es}^{15}$ confirms early clot regression in acute $\mathrm{PE}$ following 21 days of rivaroxaban in the $88 \%$ of patients with PE.

Many of the pharmacological effects of Rivaroxaban and other DOACs on cardiovascular system remain unexplored at this time. Since novel mechanisms might be involved in venous recanalization during rivaroxaban treatment, we might have to return from bedside to the bench (basic science) to understand these mechanisms. Additional data with the DOACs will emerge from basic science as well as prospective clinical trials, ultimately helping cardiovascular patients prevail over such severe diseases.

We consider that our results showed that Rivaroxaban could have a fibrinolytic effect not only on recent thrombus, as demonstrated from several trials, but also on organized thrombus with, as result, a complete recanalization of the previous residual thrombus. This action probably is due to the effect on remodeling of endothelium, considering that the organization of thrombus begins after adherence of the clot to the vessel wall, with formation of a thin lining of endothelial cells over its surface, followed by ingrowth of cells from the intima media and capillary buds into the thrombus. 
Our data allow us to consider rivaroxaban as useful drug for reducing the incidence of post-thrombotic syndrome saving the valves structure.

It may be clinically relevant to identify the speed of clot resolution during anticoagulant treatment. As in patients with a suspected recurrent DVT, it is often unclear whether thrombi reflect a recurrence or an old thrombus. Consequently, knowledge about the time to clot resolution

\section{References}

1) McRae SJ, Ginsberg JS Initial treatment of venous thromboembolism. Circulation 2004; 110: I3-I9.

2) Ageno W, Gallus AS, Wittkowsky A, Crowther M, Hylek EM, Palareti G. Oral anticoagulant therapy: antithrombotic therapy and prevention of thrombosis. 9th ed: American College of Chest Physicians evidence-based clinical practice guidelines. Chest 2012; 141: e44S-e88S

3) Brummel-Ziedins KE, Orfeo T, Gissel M, Mann KG, Rosendaal FR. Factor Xa generation by computational modeling: an additional discriminator to thrombin generation evaluation. PLoSONE 2012: 7:e29178. doi: 10.1371/journal.pone.0029178

4) Bauer KA. Pros and cons of new oral anticoagulants. Hematology 2013; 7 : 465-70.56.

5) Allegra C, Antignani PL, Kalodiki E. News in Phlebology. Minerva Medica Ed. Torino 2013.

6) Garcia DA, Baglin TP, Weitz JI, Samama MM. Parenteral anticoagulants: antithrombotic therapy and prevention of thrombosis, 9thed: American College of Chest Physicians evidence-based clinical practice guide lines. Chest 2012; 141: e24S-e43S.

7) Einstein Investigators, Bauersachs R, Berkowitz SD, et al. Oral rivaroxaban for symptomatic venous thromboembolism. N Engl J Med. 2012;363(26):2499-2510.

8) Einstein-PE Investigators, Büller HR, Prins MH, et al. Oral rivaroxaban for the treatment of symptomatic pulmonary embolism. N Engl J Med. 2012;366(14):1287-1297. might be helpful in the diagnostic work-up of patients with recurrent DVT. In our case reports, we observed the complete resolution of acute thombi after 2 weeks and the complete recanalization of old thrombi after 4 weeks.

Of course, it is necessary more observation to confirm these hypotheses and to establish the correct dosage of drug but probably the single drug approach (20 mg OD) at long time could be the correct treatment.

9) Rodriguez RA, Carrier M, Wells PS. Non-adherence to new oral anticoagulants: a reason for concern during long-term anticoagulation? J Thromb Haemost. 2013;11(2):390-394.

10) Weitz JI, Gross PL. New oral anticoagulants: which one should my patient use? Hematology Am Soc Hematol Educ Program. 2012;2012:536-540.

11) Garabon JJ, Boffa MB. Impact of Thrombomodulin and Thrombin-activatable Fibrinolysis Inhibitor on the Anti-coagulant and Pro-fibrinolytic Effects of Rivaroxaban Arteriosclerosis, Thrombosis, and Vascular Biology. 2016;36:A34.

12) Álvarez E, Paradela-Dobarro B, Raposeiras-Roubín S, González-Juanatey JR Protective, repairing and fibrinolytic effects of rivaroxaban on vascular endothelium. Br J Clin Pharmacol 2018; 84: 280-291.

13) Cheung YW, Middeldorp S, Prins MH, Pap AF, Lensing AW, Ten Cate-Hoek AJ, et al. Post-thrombotic syndrome in patients treated with rivaroxaban or enoxaparin/vitamin $\mathrm{K}$ antagonists for acute deep-vein thrombosis. Apost-hoc analysis. Thromb Haemost 2016;116:733-8.

14) EINSTEIN-PE Investigators, Büller HR, Prins MH, Lensin AW, Decousus H, Jacobson BF, et al. Oral rivaroxaban for the treatment of symptomatic pulmonary embolism. N Engl J Med 2012;366:1287-97.

15) van Es $\mathrm{J}$ et al, Clot resolution after 3 weeks of anticoagulant treatment for pulmonary embolism: comparison of computed tomography and perfusion scintigraphy J Thromb Haemost 2013;11:679-685 